\title{
Review: usual blood pressure is directly related to vascular mortality throughout middle and old age
}

\author{
Lewington S, Clarke R, Qizilbash N, et al, for the Prospective Studies Collaboration. Age-specific relevance of usual blood \\ pressure to vascular mortality: a meta-analysis of individual data for one million adults in 61 prospective studies. Lancet \\ 2002;360:1903-13.
}

\section{QUESTION: In adults with no history of vascular disease, what is the age specific association between usual blood pressure and vascular mortality?}

\section{Data sources}

Studies were identified through searches of Medline and EMBASE/Excerpta Medica, hand searches of meeting extracts, and discussions with investigators.

\section{Study selection}

Prospective observational studies were included that reported data on blood pressure at baseline and cause and date of death. Studies that deliberately recruited patients with a history of stroke or heart disease were excluded.

\section{Data extraction}

Reviewers contacted investigators of selected studies to obtain individual patient data. People with a history of stroke or heart disease at baseline were excluded from the analysis. Individual data were used to estimate the main risk factor of interest: usual (ie, long term) blood pressure. Meta-analysis was used to relate mortality during each decade of death to the estimated usual blood pressure at the start of that decade; analyses were corrected for time dependent regression dilution.

Sources of funding:

UK Medical Research

Council; British Hear

Foundation;EU

Biomed Programme;

CTSU.

For correspondence: PSC Secretariat, Radcliffe Infirmary, Oxford, UK.

psc@ctsu.ox.ac.uk years, a lower usual systolic or diastolic blood pressure was associated with a lower risk of vascular death. The association was less extreme at older ages than at younger ages.

\section{Conclusion}

In people with no history of vascular disease, usual blood pressure is directly related to vascular mortality throughout middle and old age, even at pressures as low as $115 / 75 \mathrm{~mm} \mathrm{Hg}$.

\section{COMMENTARY}

The study by Lewington $e t a l$ is unique in estimating long term blood pressure and its associations with vascular mortality for an extremely large number of people. It relies on a statistical technique to correct for regression dilution bias.

What is regression dilution bias?

Let's take an example of a study to assess the risk of lung cancer over 20 years among people who say they smoke compared with non-smokers. At baseline, it is likely that some people who report not smoking do in fact smoke and vice versa. In addition, over the follow up period, people will stop and start smoking. For simplicity, let's say the true effect of long term smoking is to increase the risk of lung cancer tenfold. In our study, we will actually measure the risk of lung cancer among people, most of whom smoked for some of the 20 years, and compare this with the risk among people, most of whom did not smoke for some of the 20 years. The inaccurate measurement of exposure to smoking has made our 2 comparison groups more similar to each other. The observed risk among our "smoking" group is likely to be considerably less than 10 times the risk among our "non-smokers". We have "diluted" our comparison groups, leading to bias in the estimate of the regression slope between our exposure (smoking) and our outcome (lung cancer) - hence the name "regression dilution bias".

Blood pressure fluctuates with time. Analyses based on a single baseline reading will tend to underestimate the effect of blood pressure on subsequent health outcomes. One way to overcome this regression dilution bias is to base our analyses on participants' "usual" or long term average blood pressure. A proportion of people included in this metaanalysis had repeated blood pressure measurements over time, allowing the authors to estimate people's "usual" blood pressure, and thus correct for regression dilution bias. This correction itself is an inexact process. However, the rigorous methods used should have ensured that this review provided the most accurate estimates to date of the effects of blood pressure on subsequent health outcomes. From 40-89 years of age, and for blood pressure $>155 / 75 \mathrm{~mm} \mathrm{Hg}$, the risk of vascular mortality is clearly increased for any increment in systolic or diastolic blood pressure.

Liam Smeeth, MBChB London School of Hygiene E̋ Tropical Medicine London, UK 